\title{
An accurate diagnosis of odontogenic cutaneous sinus tract by different computed tomography unit setting
}

\author{
Buyanbileg Sodnom-Ish*, Mi Young Eo*, Soung Min Kim \\ Department of Oral and Maxillofacial Surgery, Dental Research Institute, School of Dentistry, Seoul National University, Seoul, Korea
}

\begin{abstract}
J Korean Assoc Oral Maxillofac Surg 2021;47:51-56)
Due to their rarity and the lack of associated dental symptoms, odontogenic cutaneous sinus tracts (OCSTs) are often misdiagnosed and confused with cutaneous lesions or non-odontogenic infections. It has been estimated that $50 \%$ of individuals affected by OCSTs are subjected to inappropriate treatments before the correct diagnosis is established. We describe the diagnosis and treatment of two cases of OCSTs. By using a computed tomography (CT) with soft tissue window setting, the extent of cortical bone destruction and the path of the sinus tract in the soft tissue was easily identified. Thus, we recommend the use of imaging techniques such as $\mathrm{CT}$, which can confirm the odontogenic origin and the exact location of the OCST.
\end{abstract}

Key words: Cutaneous fistula, Computed tomography, Periapical abscess

[paper submitted 2020. 8. 26 / revised 2020.11. 1 / accepted 2020. 11. 2]

\section{Introduction}

Odontogenic cutaneous sinus tracts (OCSTs), or cutaneous fistulas of dental origin, are rare dermatoses that occur because of chronic dental draining infections ${ }^{1}$. It has been estimated that $50 \%$ of the individuals affected by OCSTs are subjected to inappropriate dermatological treatments and an array of antibiotic treatments before the correct diagnosis is established ${ }^{2}$. The diagnosis of OCST can be challenging because of the anatomic complexity of the oral cavity and the asymptomatic nature of chronic dental infections. Correct diagnosis often requires a variety of methods (e.g., radiological, microbiological, and/or pathological).

Patients with OCST often seek initial medical help from a general surgeon or a dermatologist instead of a dentist or

\footnotetext{
Soung Min Kim

Department of Oral and Maxillofacial Surgery, Dental Research Institute, School of Dentistry, Seoul National University, 101 Daehak-ro, Jongno-gu, Seoul 03080, Korea

TEL: +82-2-2072-0213

E-mail:smin5@snu.ac.kr

ORCID: https://orcid.org/0000-0002-6916-0489

*These authors contributed equally to this work as first authors.

(c) This is an open-access article distributed under the terms of the Creative Commons Attribution Non-Commercial License (http://creativecommons.org/ licenses/by-nc/4.0/), which permits unrestricted non-commercial use, distribution, and reproduction in any medium, provided the original work is properly cited. Copyright (C) 2021 The Korean Association of Oral and Maxillofacial Surgeons. All rights reserved.
}

a maxillofacial surgeon and undergo multiple surgical excisions, radiotherapy, biopsies, and antibiotic regimens with eventual recurrence because the primary odontogenic cause is not diagnosed ${ }^{3}$. Thus, a diagnosis made with suspicion of odontogenic origin, appropriate examination methods, and prompt treatment will reduce or eliminate complications such as asepsis, osteomyelitis, and patient discomfort ${ }^{4}$. In this article, we present our experience utilizing computed tomography $(\mathrm{CT})$ with the soft tissue window setting to confirm suspected OCST and to visualize the sinus tract.

\section{Cases Report}

\section{Case 1}

A 27-year-old man presented with a chief complaint of a draining lesion on his left submandibular region with pus extrusion. Previously, the patient had undergone irrigation and drainage treatment by a general surgeon. The patient also disclosed that after the irrigation and the drainage treatment, intermittent pus discharge from the lesion was observed without pain. He had also visited a local dental clinic and extraction of his infected second molar tooth was recommended. The patient had no significant medical history with the exception of smoking one cigarette per day.

Clinical examination revealed a non-tender, erythematous 
nodule measuring about $0.7 \mathrm{~cm}$ in diameter on the left submandibular region.(Fig. 1. A) Upon gentle palpation pus discharge from the lesion was observed. Intra-oral examination was unremarkable. Panoramic radiogram revealed periapical rarefaction of the \#31, \#37, and \#47 teeth.(Fig. 1. B) Coronal, sagittal, and axial CT optimized with 45 Hounsfield unit (HU) window length (WL) and $300 \mathrm{HU}$ window width (WW) demonstrated a perforation of the cortical plate of the \#37 tooth and a fistulous tract with high attenuation connected to the cutaneous layer.(Fig. 1. C-E)

Based on these findings, the proposed treatment plan of fistulectomy of the left lower buccal cheek and mucosa, mass excision of the right and left molar region for biopsy and surgical extraction of the diseased $\# 37$, \#47 teeth along with the impacted \#38, \#48 third molar teeth were carried out under conscious sedation with $4 \mathrm{mg}$ midazolam. The biopsy results showed periapical granuloma from the periapical area of the \#37 tooth and chronic periodontitis from the periapical area of the \#47 tooth. Wound healing proceeded uneventfully. Significant improvements were observed at the one-year followup.

\section{Case 2}

In January 2018, a 59-year-old woman was referred to confirm the dental origin of a skin lesion. The patient had a chief complaint of the presence of a nonhealing extraoral nodule and a persistent abscess that had started three months prior. The patient was initially referred to a dermatologist and a general surgeon in a local hospital prior to the visit.
Extraoral examination revealed an erythematous, nontender nodule with crusting approximately $1 \mathrm{~cm}$ in diameter slightly above the inferior border of the right mandible.(Fig. 2. A) Intra-oral examination revealed gold crown restorations on the \#45, \#46, and \#47 teeth. The panoramic radiogram revealed a periapical rarefaction of the \#45 tooth with an incomplete endodontic treatment status. Coronal, sagittal, and axial CT using the bone setting showed a perforation of the cortical bone of the \#45 tooth and mild swelling of the adjoining buccal soft tissue.(Fig. 2. B-D) However, a cutaneous sinus tract could not be detected on the bone setting of the CT with a 3,000 HU WW and a $400 \mathrm{HU}$ WL. After optimizing the window/level transformation to the soft tissue setting of $250 \mathrm{HU}$ WW and $50 \mathrm{HU}$ WL, an OCST connecting the affected tooth to the subcutaneous layer of the skin was delineated on the axial, coronal, and sagittal views of the scan.(Fig. 2. E-G)

Cutaneous fistula closure on the right cheek and removal of the \#45 tooth were performed without any complications. After ten months, the OCST on the right cheek healed completely and the scar was almost imperceptible.(Fig. 3. A) Dental implant installation for the edentulous area of the $\# 45$ tooth was carried out. A guided bone regeneration technique was applied to cover the bone defect using allogenic Oragraft (LifeNet Health, Virginia Beach, VA, USA). A 4.0×8.5-mm internal submerged-type Luna fixture (Shinhung, Seoul, Korea) was then inserted.

There were no remarkable findings at the four-month follow-up. Re-entry surgery was performed at that time under local anesthesia, and a limited incision was made to expose

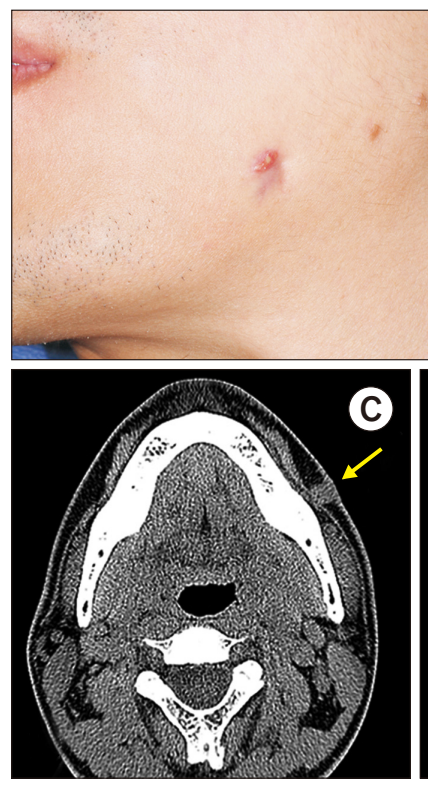

(A)

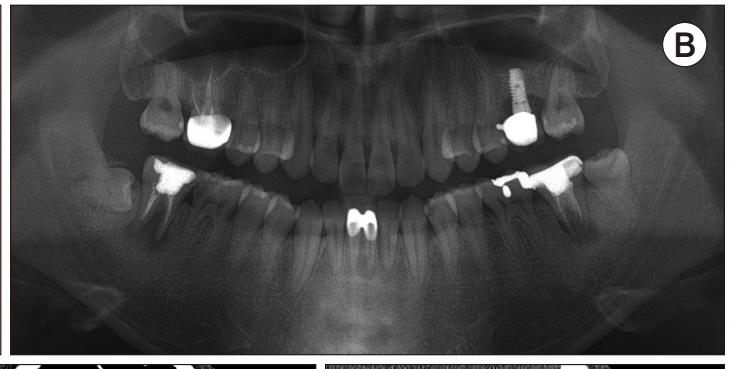

Fig. 1. A. Clinical examination revealed a non-tender, erythematous nodule measuring approximately $0.7 \mathrm{~cm}$ in

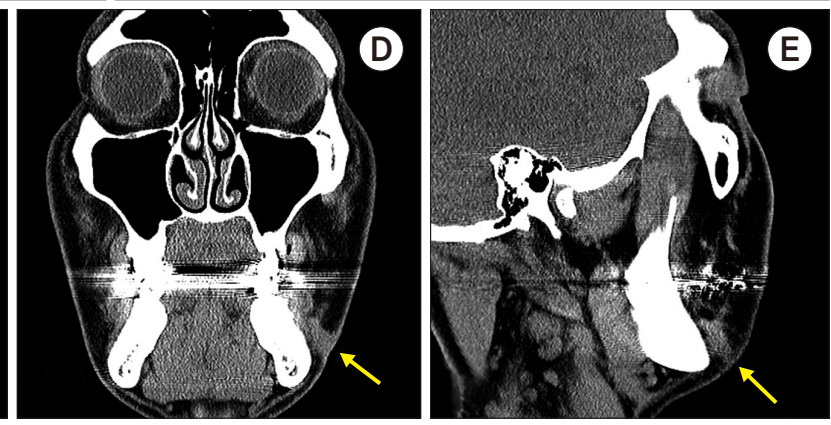
diameter on the left submandibular region. B. Preoperative panoramic view revealed a periapical rarefaction of the \#31, \#37, \#47 teeth and impacted \#38, \#48 third molar teeth. C-E. Axial, coronal, and sagittal computed tomography showing the exact path of the odontogenic sinus tract marked with arrows. Buyanbileg Sodnom-Ish et al: An accurate diagnosis of odontogenic cutaneous sinus tract by different computed tomography unit setting. J Korean Assoc Oral Maxillofac Surg 2021 

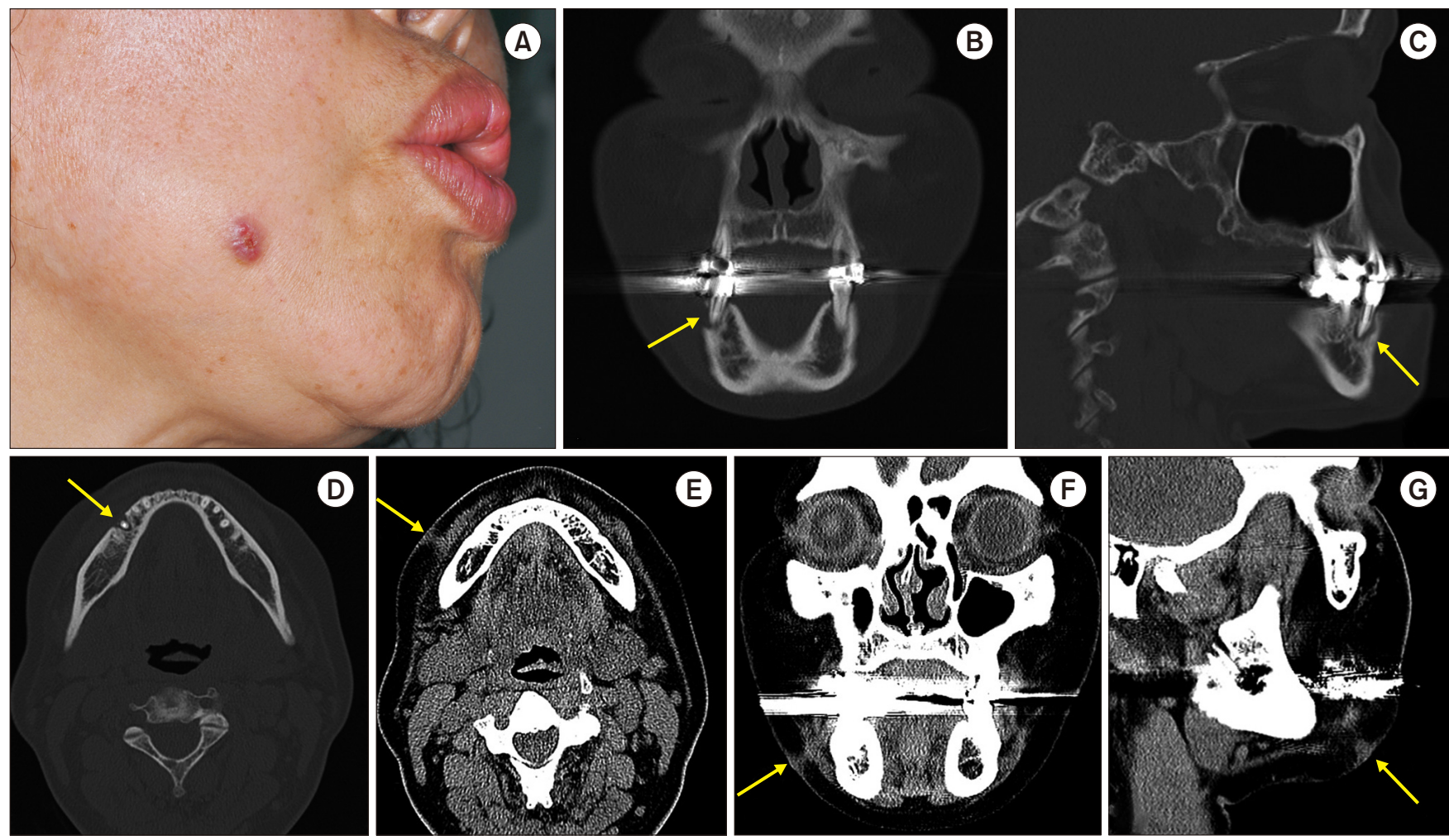

Fig. 2. A. Extraoral view of a patient with an orocutaneous fistula in the right mandible cheek. B-D. Preoperative axial, coronal, and sagittal computed tomography (CT) reveal lingual bone destruction of the \#45 tooth and mild swelling of the adjoining buccal soft tissue marked with arrows. E-G. Axial, coronal, and sagittal CT with the soft tissue setting demonstrate the origin and the route of the fistula connected to the subcutaneous layer of the skin marked with arrows.

Buyanbileg Sodnom-Ish et al: An accurate diagnosis of odontogenic cutaneous sinus tract by different computed tomography unit setting. J Korean Assoc Oral Maxillofac Surg 2021
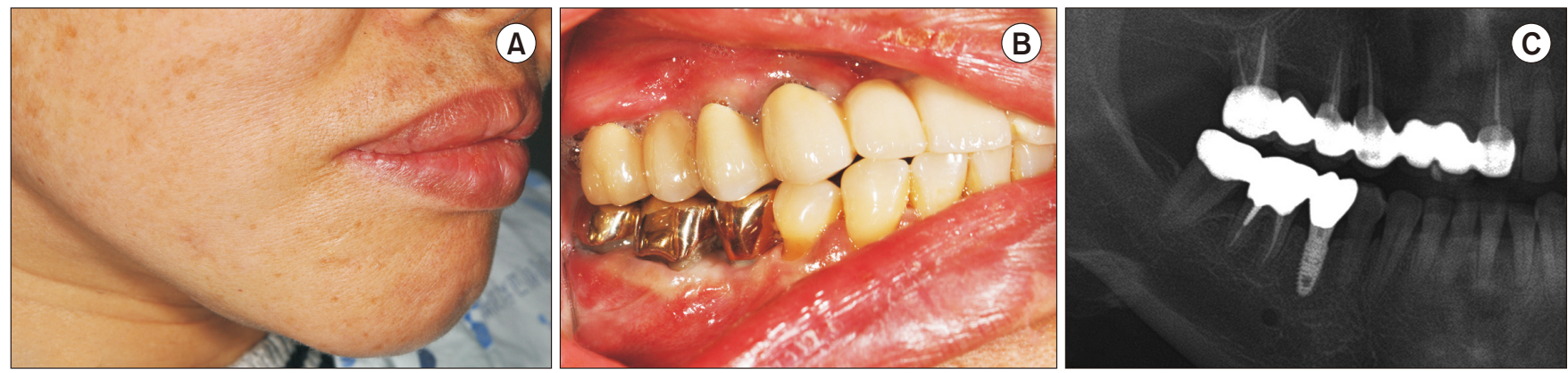

Fig. 3. A. Postoperative clinical photo of nearly imperceptible scarring after ten months. B. Clinical view of the final prosthesis delivery. C. Panoramic radiogram showing a well-osseointegrated implant along with prosthetic delivery at the 7-month follow-up after implant installation.

Buyanbileg Sodnom-Ish et al: An accurate diagnosis of odontogenic cutaneous sinus tract by different computed tomography unit setting. J Korean Assoc Oral Maxillofac Surg 2021

the implants for healing abutment placement. Three months after re-entry surgery, the final prosthesis was placed.(Fig. 3. B) Panoramic radiogram seven months after implant installation showed a well-osseointegrated implant placed in the right mandible.(Fig. 3. C)

\section{Discussion}

A dental etiology should always be considered in the differential diagnosis of any orofacial skin lesion. A cutaneous sinus tract of the face and neck region is usually caused by dental infection. Other causes may include osteomyelitis, actinomycosis, pyogenic granuloma, neoplasms, salivary gland fistula, infected cyst, deep mycotic infection, and other 
local skin infections (e.g., carbuncles and infected epidermoid cysts) ${ }^{3,5,6}$. A delay in the treatment of such infections can cause the infection to spread into the fascial spaces of the orofacial area or into the deep spaces of the head and neck region (parapharyngeal space infection) ${ }^{3}$.

Most cutaneous sinus tracts have an intraoral opening, but in cases of chronic dental infection, the local inflammatory process progresses slowly as an alveolar bone abscess, following the path of least resistance through the maxillary or mandibular periosteum. The site of the opening depends on the location of the bony perforation in relation to the facial muscle attachments. In the mandible, if the osseous perforation occurs above the muscle attachment, the fistulae may occur in the attached mucosa, the buccal vestibule, or the floor of the mouth. If the osseous perforation is located below the muscle attachments, the sinus will open extraorally and appear in the chin, submental, or submandibular region ${ }^{4}$. In our first and the second case, the erythematous nodule presented slightly above the inferior border of the mandible.

Pulp sensitivity test, tracking the dental origin of the sinus tract with the gutta-percha point, and radiographic examination have been for cutaneous sinus tract evaluation ${ }^{1,5,7}$. However, the pulp sensitivity test may determine the condition of the teeth but fail to identify the path of the sinus tract. Furthermore, the gutta-percha technique may determine the direction and the depth of the sinus tract, the possible presence of a foreign body, and communication of fistula with the apices of teeth, but it is a blind procedure, and panoramic radiography may result in a false diagnosis due to overlapping. Panoramic radiography is often used for diagnosing cutaneous sinus fistulae of dental origin, but it cannot determine the exact extent of bone lesions and soft tissue pathology associated with the sinus tract. Cone-beam computed tomography (CBCT) has been the recommended imaging modality for evaluating the size and extent of bone destruction around the apices and locating the tooth involved in cutaneous sinus tract cases ${ }^{5}$. In our case, CT with the default bone window setting showed the exact extent of cortical bone destruction; by adjusting the window setting to soft tissue, the path of the sinus tract in the soft tissue could then be identified.

The sinus tracts found in the head and neck region are mainly caused by odontogenic infections, taking up $80 \%$, while the remaining $20 \%$ is caused by non-odontogenic origin. The major causes of odontogenic sinus tracts include periapical and periodontal infection $(63.5 \%)$, pericoronitis resulting from mandibular teeth $(8.5 \%)$, fracture mandible $(7 \%)$, infected dentigerous/keratocyst $(6 \%)^{8}$. Therefore, it is impor- tant to have a high suspicion of odontogenic origin in the first place for cutaneous sinus tracts found in the head and region. Other major causes, which have been confused with the dermal origin is osteomyelitis and other congenital causes, eg, parotid, thyroglossal, or branchial ${ }^{9}$. For correct differential diagnosis, sinus tracts found in osteomyelitis will be identified with our method along with osteolysis, sequestra formation, and sclerosing lesions in CT findings ${ }^{10}$ allow identify the obvious origin of the sinus tract. In parotid sinus tracts found in the head and neck region, the CT findings will also allow visualizing the abnormal congenital soft tissue findings such as ectopic parotid gland position allowing to differentiate from other origins ${ }^{11}$.

Furthermore, the developmental route of the odontogenic cutaneous sinus tract is also an important factor to consider for correct differential diagnosis. The inflammatory process causing periapical abscess progresses slowly resorbing the bone and spreads toward the cortical plate following the path of least resistance. If the location of perforation is located above the muscle attachment including the buccinator muscle in the maxilla and mentalis, mylohyoid, and buccinator muscles in the mandible, then it is most likely that the sinus tract will drain extra-orally. If the perforation is located below the mentioned muscle attachments, the sinus tract is most likely to drain intra-orally ${ }^{7}$. Thus, in clinical examination, if the orifice is identified externally above the muscles, the dermal origin should be considered and CT analysis with soft tissue setting will help to verify the diagnosis.

The primary objective for managing OCST is to remove the source of infection which may be eliminated by removal of the infected pulp tissue through endodontic treatment or, if there is no indication for endodontic treatment or apical resection, by extraction of the infected tooth and curettage ${ }^{1}$. Once the odontogenic cause is removed, the OCST will heal without further treatment within a few weeks as our cases' healing uneventfully ${ }^{12}$. Accordingly, our cutaneous sinus tract cases resolved uneventfully after elimination of the source of infection.

Coronal, sagittal, and axial CT adjusted with abdomen setting, such as $45 \mathrm{HU}$ WL and $300 \mathrm{HU}$ WW in the INFINITT PACS software (INFINITT Healthcare, Seoul, Korea), demonstrated a perforation of the cortical plate of the \#37 and \#45 tooth with a high attenuated fistulous tract connected to the cutaneous layer in each case.(Fig. 3) After optimizing the WL/WW transformation to the abdomen setting of 45-50 HU WL and 250-300 HU WW, any OCST connecting the affected tooth to the subcutaneous layer of the skin was delineated 
clearly with mild swelling of the adjoining buccal soft tissue on the axial, coronal, and sagittal views. Panoramic radiography and CBCT were often used to visualize the exact location of spatial periapical radiolucency and the cortical plate perforation, but it demonstrates a poor diagnostic potential with soft tissue lesions due to limited contrast resolution ${ }^{5,13-15}$. This proposed method can be implemented with any other types of commercially available picture archiving and communications systems (PACS) such as the M-view (Marosis M-view 4.5; Marotech, Seoul, Korea), Philips DiagNET 2.2 software (Philips, Amsterdam, The Netherlands) ${ }^{16}$, PACSPLUS viewer 3.2 (Pacsplus, Orange, CA, USA) ${ }^{17}$, dicomPACS DX-R (OR Technology, Rostock, Germany) ${ }^{18}$ or Digital Imaging and Communications in Medicine (DICOM) viewing software RadiAnt DICOM 2.2.9 viewer (Medixant Company, Poznań, Poland $)^{19}$. The application system of these programs allows medical practitioners to adjust WL/WW values manually, focusing on the visibility of tissues relevant to the pathology. Recently, any PACS programs used in the clinical practice has the pre-set WL/WW values such as the "abdomen", "angio", "bone", "brain", "chest", and "lungs" configurated for analyzing the relevant tissues. Therefore, this method can be used universally in any clinical practice with PACS system, which will allow an easy and convenient way to validate the exact route and the dental origin of the OCSTs.

In conclusion, the use of CT with the WL/WW values adjusted for bone and soft tissue evaluation, allows clinicians to determine the exact source of infection, the route of medullary and cortical bone perforation, and soft tissue changes of OCSTs. Thus, use of different WL/WW values make timely and accurate diagnosis, avoiding time-consuming, unnecessary additional diagnostic techniques in the facial dermatologic management.

\section{ORCID}

Buyanbileg Sodnom-Ish, https://orcid.org/0000-00024239-1420

Mi Young Eo, https://orcid.org/0000-0001-7055-9924

Soung Min Kim, https://orcid.org/0000-0002-6916-0489

\section{Authors' Contributions}

B.S.I. participated in data collection and wrote the manuscript. M.Y.E. participated in the study design and coordination and helped to draft the manuscript. S.M.K. read and approved the final manuscript.

\section{Acknowledgements}

This study was supported by Basic Science Research Program through the National Research Foundation of Korea (NRF) funded by the Ministry of Education (2017R1D1A1B04029339).

\section{Ethics Approval and Consent to Participate}

This article was approved by the Institutional Review Board of Seoul National University (S-D20200010), and informed consent was obtained from the individual patient included in this study.

\section{Consent for Publishing Photographs}

Written informed consent was obtained from the patients for publication of this article and accompanying images.

\section{Conflict of Interest}

No potential conflict of interest relevant to this article was reported.

\section{References}

1. Guevara-Gutiérrez E, Riera-Leal L, Gómez-Martínez M, AmezcuaRosas G, Chávez-Vaca CL, Tlacuilo-Parra A. Odontogenic cutaneous fistulas: clinical and epidemiologic characteristics of 75 cases. Int J Dermatol 2015;54:50-5. https://doi.org/10.1111/ijd.12262

2. Pasternak-Júnior B, Teixeira CS, Silva-Sousa YT, Sousa-Neto MD. Diagnosis and treatment of odontogenic cutaneous sinus tracts of endodontic origin: three case studies. Int Endod J 2009;42:271-6. https://doi.org/10.1111/j.1365-2591.2008.01519.x

3. Mittal N, Gupta P. Management of extra oral sinus cases: a clinical dilemma. J Endod 2004;30:541-7. https://doi. org/10.1097/00004770-200407000-00019

4. Mukerji R, Jones DC. Facial sinus of dental origin: a case report. Dent Update 2002;29:170-1. https://doi.org/10.12968/ denu.2002.29.4.170

5. Tian J, Liang G, Qi W, Jiang H. Odontogenic cutaneous sinus tract associated with a mandibular second molar having a rare distolingual root: a case report. Head Face Med 2015;11:13. https://doi. org/10.1186/s13005-015-0072-y

6. Gupta M, Das D, Kapur R, Sibal N. A clinical predicament--diagnosis and differential diagnosis of cutaneous facial sinus tracts of dental origin: a series of case reports. Oral Surg Oral Med Oral Pathol Oral Radiol Endod 2011;112:e132-6. https://doi.org/10.1016/ j.tripleo.2011.05.037

7. Sammut S, Malden N, Lopes V. Facial cutaneous sinuses of dental origin - a diagnostic challenge. Br Dent J 2013;215:555-8. https:// doi.org/10.1038/sj.bdj.2013.1141

8. Kishore Kumar RV, Devireddy SK, Gali RS, Chaithanyaa N, Chakravarthy C, Kumarvelu C. Cutaneous sinuses of cervicofacial region: a clinical study of 200 cases. J Maxillofac Oral Surg 2012;11:411-5. https://doi.org/10.1007/s12663-012-0353-y 
9. Chowdri NA, Sheikh S, Gagloo MA, Parray FQ, Sheikh MA, Khan FA. Clinicopathological profile and surgical results of nonhealing sinuses and fistulous tracts of the head and neck region. J Oral Maxillofac Surg 2009;67:2332-6. https://doi.org/10.1016/ j.joms.2008.06.084

10. Kim SM, Lee SK. Chronic non-bacterial osteomyelitis in the jaw. J Korean Assoc Oral Maxillofac Surg 2019;45:68-75. https://doi. org/10.5125/jkaoms.2019.45.2.68

11. Moon WK, Han MH, Kim IO, Sung MW, Chang KH, Choo SW, et al. Congenital fistula from ectopic accessory parotid gland: diagnosis with CT sialography and CT fistulography. AJNR Am J Neuroradiol 1995;16(4 Suppl):997-9.

12. Shemesh A, Hadad A, Azizi H, Lvovsky A, Ben Itzhak J, Solomonov M. Cone-beam computed tomography as a noninvasive assistance tool for oral cutaneous sinus tract diagnosis: a case series. J Endod 2019;45:950-6. https://doi.org/10.1016/j.joen.2019.03.017

13. Angelopoulos C, Scarfe WC, Farman AG. A comparison of maxillofacial CBCT and medical CT. Atlas Oral Maxillofac Surg Clin North Am 2012;20:1-17. https://doi.org/10.1016/ j.cxom.2011.12.008

14. Miracle AC, Mukherji SK. Conebeam CT of the head and neck, part 1: physical principles. AJNR Am J Neuroradiol 2009;30:108895. https://doi.org/10.3174/ajnr.A1653

15. Weiss R 2nd, Read-Fuller A. Cone beam computed tomography in oral and maxillofacial surgery: an evidence-based review. Dent $\mathrm{J}$
(Basel) 2019;7:52. https://doi.org/10.3390/dj7020052

16. MDC PACS viewer user guide R2.3 SP2 [Internet]. Amsterdam: Philips [cited 2020 Nov 1]. Available from: https://home.itp. ac.ru/ alexd/DiagNET/Help/MDC_PACS_Viewer_UM_R2.3SP2_ ENGLISH.pdf

17. Medlink PACS Plus viewer user manual [Internet]. Goleta (CA): iCRco [cited 2020 Nov 1]. Available from: https://vdocuments.mx/ pacs-plus-viewer-user-manual.html

18. Acquisition and diagnostic software for $X$-ray images from $D R$ flat panels or CR systems [Internet]. Rostock: OR Technology [cited 2020 Nov 1]. Available from: https://www.or-technology.com/media/downloads/Brochure\%20dicomPACS\%20DX-R_EN.pdf

19. RadiAnt DICOM Viewer [Internet]. Poznań: Medixant [cited 2020 Nov 1]. Available from: https://www.radiantviewer.com/dicomviewer-manual/change_brightness_contrast.html

How to cite this article: Sodnom-Ish B, Eo MY, Kim SM. An accurate diagnosis of odontogenic cutaneous sinus tract by different computed tomography unit setting. J Korean Assoc Oral Maxillofac Surg 2021;47:51-56. https://doi.org/10.5125/jkaoms.2021.47.1.51 